\title{
Extension of Some Common Fixed Point Theorems
}

\author{
Krishna P. Patel and G. M. Deheri
}

\begin{abstract}
It is well-known that the classical Banach's fixed point theorem has been generalized in various directions. This celebrated result has been extended to the theory of common fixed points. A few theorems dealing with the existence and uniqueness of common fixed points are presented here. These results not only extend but also sharpens some of the well known results found in the literature. There is a result which says that there does exist an improvement in the rate of convergence also.
\end{abstract}

Index Terms-Banach fixed point theorem, common fixed point, hilbert space, uniqueness.

\section{INTRODUCTION}

\section{A. Definition 1.5: Fixed Point}

Let $E$ be a metric space and $T: E \rightarrow E$ be a mapping. A point $x$ is said to be a fixed point of $T$ if $T(x)=x$.

The well-known classical result of Banach's fixed point Theorem has been extended and generalized in various directions. In the year 1922 Banach obtained the existence and uniqueness of the fixed point.

Banach Fixed Point Theorem:

Let $(X, d)$ be a complete metric space and $T: X \rightarrow X$ satisfying

$$
d(T(x), T(y)) \leq k d(x, y) \quad \forall x, y \in X
$$

and for some $k$ with $0<k<1$, then $T$ has a unique fixed point in $X$.

This result was generalized in some direction by Browder and Petryshyn [1]

\section{B. Definition 1.6: Common Fixed Point}

Let $H$ be a Hilbert space and $S, T: H \rightarrow H$ be two mappings. A point $x$ is said to be a common fixed point of $S$ and $T$ if $T(x)=x=S(x)$.

Koparde and Waghmode [2] extended the result of Jungck [3] and Fisher [4] to common fixed points. Here, we proceed to extend and sharpen the main result of Koparde and Waghmode [2] by modifying their procedure.

Jungck [3] obtained the following:

Theorem 1: Let $f$ be a continuous mapping of a complete metric space $(X, d)$ into itself. Then $f$ has a fixed point in $X$ if there exists $\alpha \in(0,1)$ and a mapping $g: X \rightarrow X$ which commutes with $f$ and satisfies $g(X) \subset f(X)$ and

Manuscript received February 7, 2013; revised June 25, 2013

The authors are with the Sardar Patel University, Vallabh Vidhya Nagar, Gujarat, India (e-mail: krishnappatel10@yahoo.com, gm.deheri@rediffmail.com).

$$
d(g(x), g(y)) \leq \alpha d(f(x), f(y)) \forall x, y \in X
$$

Indeed, $f$ and $g$ have a unique common fixed point.

Fisher [4] extended and modified the above result in the form of

Theorem 2: Let $S$ and $T$ be continuous mapping of complete metric space $(X, d)$ into itself. Then $S$ and $T$ have a common fixed point in $X$ iff there exists a continuous mapping $A$ of $X$ into $S X \cap T X$, which commute with $S$ and $T$ and satisfies the inequality

$$
d(A x, A y) \leq \alpha d(S x, T y) \quad \forall x, y \in X
$$

where $0<\alpha<1$. Indeed, $S, T$, and $A$ then have a unique common fixed point.

Extending further, Koparde and Waghmode [2] obtained the following in the setting of Hilbert space.

Theorem 3: Let $S$ and $T$ be continuous mapping of a Hilbert space $X$ into itself. Then $S$ and $T$ have a common fixed point in $X$ iff there exists a continuous mapping $A$ of $X$ into $S X \cap T X$, which commute with $S$ and $T$ and satisfies the inequality

$$
\|A x-A y\| \leq \alpha\|A x-S x\|+\beta\|A y-T y\|+\gamma\|S x-T y\|
$$

for all $x, y$ in $X$, where $\alpha, \beta, \gamma$ are non negative reals with $0<\alpha+\beta+\gamma<1$.Indeed, $S, T$, and $A$ then have a unique common fixed point.

It is well known that, (Bisht and Joshi [5]) there exist maps that have a discontinuity in their domain but which admit fixed point, for instance, one can consider Kannan [6], [7].

Bisht and Joshi [5] derived common fixed point theorems for a pair of weakly reciprocally continuous self mappings satisfying generalized contraction or Lipschitz type conditions. This investigation extended the scope of the study of common fixed point theorems from the class of compatible continuous mappings to a wider class of mappings which included noncompatible and discontinuous mappings. The following is the main result of this discussion.

Theorem 4: Let $f$ and $g$ be weakly reciprocally continuous self mappings of a complete metric space $(X, d)$ such that

(i) $f \mathrm{X} \subseteq g X$

(ii) $d(f x, f y) \leq a d(g x, g y)+b[d(f x, g x)+d(f y, g y)]$ $+c[d(f x, g y)+d(f y, g x)]$, $a, b, c \geq 0,0 \leq a+2 b+2 c<1$.

If $f$ and $g$ are either compatible or $g$-compatible or $\mathrm{f}$ compatible or compatible of type $(P)$ then $f$ and $g$ have a 
unique common fixed point.

In fact, Pant [8] introduced the concept of reciprocal continuity and applied it to establish a situation where in a collection of mappings has a fixed point, which is a point of discontinuity of all mappings.

Subsequently, a large number of papers dealt with the application of reciprocal continuity.

Towards the generalization of reciprocal continuity, Pant, Bisht, Arora [9] introduced weak reciprocal continuity and employed this new notion to obtain fixed point theorems. Indeed, this new notion was applicable to compatible mappings as well as non compatible mappings. In this investigation the following result is found to be conclusively established.

Theorem 5: Let $f$ and $g$ be weakly reciprocally continuous self mappings of a complete metric space $(X, d)$ such that

$$
\begin{gathered}
(i) f \mathrm{X} \subseteq g X \\
(i i) d(f x, f y) \leq a d(g x, g y)+b d(f x, g x) \\
+c d(f y, g y), \\
0 \leq a, b, c<1,0 \leq a+b+c<1 .
\end{gathered}
$$

If $f$ and $g$ are either compatible or R-weakly commuting of type $\left(A_{g}\right)$ or R-weakly commuting of type $\left(A_{f}\right)$ then $f$ and $g$ have a unique common fixed point.

Recently, Pant and Bisht [10] unified the approaches of reciprocal continuity, subsequential continuity and conditional commutativity to generalize the notion of reciprocal continuity. Here, some common fixed point theorems in diverse settings were obtained as an application of the new notion introduced. Of particular importance is the following result from Pant and Bisht [10].

Theorem 6: Let $f$ and $g$ be conditionally reciprocally continuous selfmappings of a complete metric space $(X, d)$ such that

$$
\text { (i) } f \mathrm{X} \subseteq g X
$$

(ii) $d(f x, f y) \leq k d(g x, g y), k \in[0,1)$.

If $f$ and $g$ are either compatible or g-compatible or $f$ compatible then $f$ and $g$ have a unique common fixed point.

Takahashi [11] introduced the notion of convexity in metric spaces, and discussed some fixed point theorems for nonexpansive mappings in such convex metric spaces.

Mohammad Moosaei [12] has studied some fixed point theorems for selfmappings satisfying certain contraction principles on a convex complete metric space. Also, he has investigated some common fixed point theorems for a Banach operator pair under certain generalized contractions on a complete metric space.

In [13], a hybrid iteration method was adopted and the strong convergence of the iteration scheme to a fixed point of nonself nonexpansive mapping was obtained in Banach spaces. The principal result dealing with the strong convergence of the Hybrid iteration scheme is the following.

Theorem 7: Let $E$ be a real Banach space with a uniformly Gateaux differentiable norm and $C$ be a nonempty weakly compact convex subset of $E$. Suppose that $T$ is a nonself nonexpansive mapping. $\left\{x_{n}\right\}$ (defined as in [13]), $\left\{\alpha_{n}\right\},\left\{\lambda_{n}\right\}$ are real number sequences in $[0,1)$ satisfying the following conditions:

1) $\left(C_{1}\right)$ and $\left(C_{2}\right)$ (as in [13]);

2) $\sum_{n=2}^{\infty} \lambda_{n}<\infty$.

If $F=K_{m n} \cap F(P T) \neq \phi$, then $\left\{x_{n}\right\}$ strongly converges to some point of $F$.

Gulnara Abduvalieva and Dmitry S. KaliuzhnyiVerbovetskyi [14] established a fixed point theorem for mappings of all sizes which respected the matrix sizes and direct sums of matrices. The conclusions were stronger if such mappings were noncommutative functions respecting matrix similarities. The main result of this paper is the following.

Theorem 8: Let $S$ be a set, and let $\Omega \subseteq S_{n c}$ respect direct sums of matrices. Define supp $\Omega:=$ $\left\{n \in \mathbb{N}: \Omega_{n} \neq \phi\right\}$. Let $f: \Omega \rightarrow \Omega$ satisfy

1) $f\left(\Omega_{n}\right) \subseteq \Omega_{n}, n \in \operatorname{supp} \Omega$;

2) $f$ respects direct sums: $f(\mathrm{X} \oplus \mathrm{Y})=f(\mathrm{X}) \oplus f(\mathrm{Y})$, $\mathrm{X}, \mathrm{Y} \in \Omega$;

3) for every $\mathrm{n} \in \operatorname{supp} \Omega$ the mapping $\left.f\right|_{\Omega_{n}}$ has a unique fixed point, $\mathrm{X}_{* n}$.

Let $d=\operatorname{gcd}\{\mathrm{n}: \mathrm{n} \in \operatorname{supp} \Omega\}$.Then

1) There exist $\mathrm{X}_{*} \in S^{d \times d}$ such that

$$
\mathrm{X}_{* n}=\bigoplus_{\alpha=1}^{n / d} \mathrm{X}_{*} ; \mathrm{n} \in \operatorname{supp} \Omega \text {. }
$$

2) If, moreover, $S=M$ is a bi-module over $R, \mathrm{O}=0 \in M$, and $f$ is a $n c$ function, then there exist a $n c$ set $\tilde{\Omega} \supseteq \Omega$ with supp $\tilde{\Omega}=\mathbb{N} d$ and a $n c$ function $\tilde{f}$ : $\tilde{\Omega} \rightarrow \tilde{\Omega}$ such that

3) $\left.\tilde{f}\right|_{\Omega}=f$;

4) For every $\mathrm{n} \in \mathbb{N} d$ the mapping $\left.\tilde{f}\right|_{\tilde{\Omega}_{n}}$ has a unique fixed point

$$
\mathrm{X}_{* n}=\bigoplus_{\alpha=1}^{n / d} \mathrm{X}_{*}
$$

Here, we make an attempt to prove an improved version of this result, which also strengthens a result of Jungck [3] and Fisher [4].

\section{MAIN Results}

Theorem 9: Let $S$ and $T$ be continuous mappings of a Hilbert space $X$ into itself. Then $S$ and $T$ have a common fixed point in $X$ iff there exists a continuous mapping $A$ of $X$ into $S X \cap T X$, which commutes with $S$ and $T$ and satisfies the inequality 


$$
\|A x-A y\|^{2} \leq \alpha\|A x-S x\|^{2}+\beta\|A y-T y\|^{2}+\gamma\|A x-S y\|^{2}+\delta\|A x-T y\|^{2}
$$

for all $x, y$ in $X$; where $\alpha, \beta, \gamma, \delta$ are non-negative reals with $0<\alpha+\beta+\gamma+\delta<1$. Indeed, $S, T$ and $A$ then have a unique common fixed point.

Proof: Firstly, we prove that the existence of such a mapping $A$ is necessary. For this suppose, $S z=z=T z$ for some $z$ in $X$.

Define a mapping $A$ of $X$ into $X$ by $A x=z$ for all $x$ in $X$. Then clearly, $A$ is continuous mapping of $X$ into $S X \cap T X$. Since, $S x, T x \in X$, for all $x \in X$ and $A x=z$, for all $x \in X$, we get

$$
A S x=z, S A x=S z=z, A T x=z, T A x=T z=z .
$$

Hence, $A$ commutes with $S$ and $T$. Now, for any $\alpha, \beta, \gamma, \delta$ with $0<\alpha+\beta+\gamma+\delta<1$, it is noticed that

$$
\begin{aligned}
\|A x-A y\|^{2} \leq & \alpha\|A x-S x\|^{2}+\beta\|A y-T y\|^{2}+\gamma\|A x-S y\|^{2} \\
& +\delta\|A x-T y\|^{2}
\end{aligned}
$$

which gives

$$
0 \leq \alpha\|z-S x\|^{2}+\beta\|z-T y\|^{2}+\gamma\|z-S y\|^{2}+\delta\|z-T y\|^{2}
$$

for all $x, y$ in $X$. This proves the existence of such a mapping $A$ is necessary.

We now prove that the condition is sufficient. For this suppose that such a mapping $A$ exists. Then we construct a sequence $\left\{x_{n}\right\}$ as follows. Let $x_{0} \in X$ be an arbitrary point.

Since $A X \subset S X$, we choose a point $x_{1}$ in $\mathrm{X}$ such that $S x_{1}=A x_{0}$

Also, $A X \subset T X$ and hence we can choose $x_{2} \in X$ such that $T x_{2}=A x_{1}$.

Continuing in this way we get a sequence $\left\{x_{n}\right\}$ as follows:

$$
S x_{2 n-1}=A x_{2 n-2}, T x_{2 n}=A x_{2 n-1} \quad n=1,2,3, \ldots
$$

We proceed to show that $\left\{A x_{n}\right\}$ is a Cauchy sequence. For this we have the inequality,

$$
\begin{aligned}
\left\|A x_{2 n}-A x_{2 n-1}\right\|^{2}= & \left\|A x_{2 n-1}-A x_{2 n}\right\|^{2} \\
& \leq \alpha\left\|A x_{2 n-1}-S x_{2 n-1}\right\|^{2}+\beta\left\|A x_{2 n}-T x_{2 n}\right\|^{2} \\
& +\gamma\left\|A x_{2 n-1}-S x_{2 n}\right\|^{2}+\delta\left\|A x_{2 n-1}-T x_{2 n}\right\|^{2} \\
& \leq \alpha\left\|A x_{2 n-1}-A x_{2 n-2}\right\|^{2}+\beta\left\|A x_{2 n}-A x_{2 n-1}\right\|^{2} \\
& +\gamma\left\|A x_{2 n-1}-A x_{2 n-1}\right\|^{2}+\delta\left\|A x_{2 n-1}-A x_{2 n-1}\right\|^{2} \\
& =\alpha\left\|A x_{2 n-1}-A x_{2 n-2}\right\|^{2}+\beta\left\|A x_{2 n}-A x_{2 n-1}\right\|^{2}
\end{aligned}
$$

which gives

$$
\left\|A x_{2 n}-A x_{2 n-1}\right\|^{2} \leq \frac{\alpha}{1-\beta}\left\|A x_{2 n-1}-A x_{2 n-2}\right\|^{2}
$$

Further, it is seen that

$$
\begin{aligned}
\left\|A x_{2 n+1}-A x_{2 n}\right\|^{2} & =\left\|A x_{2 n}-A x_{2 n+1}\right\|^{2} \\
& \leq \alpha\left\|A x_{2 n}-S x_{2 n}\right\|^{2}+\beta\left\|A x_{2 n+1}-T x_{2 n+1}\right\|^{2} \\
& +\gamma\left\|A x_{2 n}-S x_{2 n+1}\right\|^{2}+\delta\left\|A x_{2 n}-T x_{2 n+1}\right\|^{2}
\end{aligned}
$$

which implies that

$$
\left\|A x_{2 n+1}-A x_{2 n}\right\|^{2} \leq \frac{\alpha}{1-\beta}\left\|A x_{2 n}-A x_{2 n+1}\right\|^{2}
$$

Since $0<\alpha+\beta+\gamma+\delta<1$, we find that if $\frac{\alpha}{1-\beta}=\lambda$ then $0<\lambda<1$.

Therefore, from (1) and (2), it is concluded that

$$
\begin{aligned}
\left\|A x_{n+1}-A x_{n}\right\|^{2} & \leq \lambda\left\|A x_{n}-A x_{n-1}\right\|^{2} \\
& \leq \lambda^{2}\left\|A x_{n-1}-A x_{n-2}\right\|^{2} \\
& \cdots \cdots \cdots \cdots \\
& \leq \lambda^{n}\left\|A x_{1}-A x_{0}\right\|^{2}
\end{aligned}
$$

Now, it follows that $\left\{A x_{n}\right\}$ is a Cauchy sequence and so it has a limit $x$ in $X$. Since sequences of $\left\{S x_{2 n+1}\right\}$ and $\left\{T x_{2 n}\right\}$ are subsequences of $\left\{A x_{n}\right\}$, they have the same limit $z$. As, $S$ and $A$ are commuting mapping, we can have

$$
\begin{aligned}
S z & =\lim _{n \rightarrow \infty} S A x_{2 n+1} \\
& =\lim _{n \rightarrow \infty} A S x_{2 n+1}=A z
\end{aligned}
$$

Similarly, we get

This gives us,

$$
T z=A z
$$

$$
T z=A z=S z
$$

It now follows that

$$
\begin{aligned}
\|A z-A A z\|^{2} \leq & \alpha\|A z-S z\|^{2}+\beta\|A A z-T A z\|^{2} \\
& +\gamma\|A z-S A z\|^{2}+\delta\|A z-T A z\|^{2}
\end{aligned}
$$

From (3) and commutativity of A with S and T, we obtain that

$$
\|A A z-T A z\|=\|A A z-A T z\|=\|A A z-A A z\|=0
$$

and

$$
\|A z-S A z\|=\|A z-A A z\| .
$$

Also, it is clear that 


$$
\|A z-T A z\|=\|A z-A A z\|
$$

Making use of these, we arrive at

$$
\|A z-A A z\|^{2} \leq(\gamma+\delta)\|A z-A A z\|^{2}
$$

Since, $\gamma+\delta<1$, we have that $A z=A A z$.

Finally, taking $A z=z_{1}$, we find that

Similarly,

$$
A z_{1}=A A z=A z=z_{1}
$$

$$
T z_{1}=T A z=A T z=A A z=A z=z_{1} \text { and } S z_{1}=z_{1}
$$

So, $z_{l}$ is a fixed point of $S, T$ and $A$.

Next, to show uniqueness of this common fixed point, let us suppose that $z_{2}$ is also a common fixed point of $S, T$ and $A$ other than $\mathrm{z}_{l}$.Then

$$
S z_{2}=z_{2}, T z_{2}=z_{2}, A z_{2}=z_{2}
$$

and also $\left\|z_{1}-z_{2}\right\| \neq 0$.

Hence, it follows that

$$
\begin{aligned}
\left\|z_{1}-z_{2}\right\|^{2} & =\left\|A z_{1}-A z_{2}\right\|^{2} \\
& \leq \alpha\left\|A z_{1}-S z_{1}\right\|^{2}+\beta\left\|A z_{2}-T z_{2}\right\|^{2} \\
& +\gamma\left\|A z_{1}-S z_{2}\right\|^{2}+\delta\left\|A z_{1}-T z_{2}\right\|^{2} \\
& \leq \alpha\left\|z_{1}-z_{1}\right\|^{2}+\beta\left\|z_{2}-z_{2}\right\|^{2} \\
& +\gamma\left\|z_{1}-z_{2}\right\|^{2}+\delta\left\|z_{1}-z_{2}\right\|^{2} \\
& =(\gamma+\delta)\left\|z_{1}-z_{2}\right\|^{2}
\end{aligned}
$$

which yields

$$
\left\|z_{1}-z_{2}\right\|^{2}=(\gamma+\delta)\left\|z_{1}-z_{2}\right\|^{2}
$$

which is a contradiction as $\gamma+\delta<1$.

Hence, uniqueness occurs.

Theorem 10: Let $S$ and $T$ be continuous mappings of a Hilbert space $X$ into itself. Then $S$ and $T$ have a common fixed point in $X$ iff there exists a continuous mapping $A$ of $X$ into $S X \cap T X$, which commutes with $S$ and $T$ and satisfies the inequality

$$
\begin{aligned}
\|A x-A y\|^{2} \leq & \alpha\|A x-S x\|\|A y-S y\|+\beta\|A x-T x\|\|A y-T y\| \\
& +\gamma\|x-y\|^{2}
\end{aligned}
$$

for all $x, y$ in $X$; where $\alpha, \beta, \gamma$ are non-negative reals with $\alpha+\beta+\gamma<1$.

Proof: First of all we prove that the existence of such a mapping $A$ is necessary. For this suppose, $S z=z=T z$ for some $z$ in $X$.

Define a mapping $A$ of $X$ into $X$ by $A x=z \forall x \in X$. Then, clearly, $A$ is a continuous mapping of $X$ into $S X \cap T X$. Since, $S x, T x \in X$ for all $x \in X$ and $A x=z$, for all $x \in X$, one get

$$
A S x=z, S A x=S z=z, A T x=z, T A x=T z=z .
$$

Hence, $A$ commutes with $S$ and $T$. Now, for any $\alpha, \beta, \gamma$ with $0<\alpha+\beta+\gamma<1$, it is noticed that

$$
\begin{aligned}
\|A x-A y\|^{2} \leq & \alpha\|A x-S x\|\|A y-S y\|+\beta\|A x-T x\|\|A y-T y\| \\
& +\gamma\|x-y\|^{2}
\end{aligned}
$$

which gives,

$$
0 \leq \alpha\|A x-S x\|\|A y-S y\|+\beta\|A x-T x\|\|A y-T y\|+\gamma\|x-y\|^{2}
$$

for all $x, y$ in $X$.

This proves the necessity of such a mapping $A$.

To prove the sufficient part, we construct a sequence $\left\{x_{n}\right\}$ as follows. Let $x_{0} \in X$ be an arbitrary point. Since $A X \subset S X$, we choose a point $x_{1}$ in $X$ such that $S x_{1}=A x_{0}$.

Also, $A X \subset T X$ and hence we can choose $x_{2} \in X$ such that $T x_{2}=A x_{1}$.

Continuing in this way, we get a sequence $\left\{x_{n}\right\}$ as follows:

$$
S x_{2 n-1}=A x_{2 n-2}, T x_{2 n}=A x_{2 n-1} \quad n=1,2,3, \ldots
$$

We proceed to show that $\left\{A x_{n}\right\}$ is a Cauchy sequence. For this one arrive at the inequality,

$$
\begin{aligned}
& \left\|A x_{2 n+1}-A x_{2 n}\right\|^{2} \\
& \leq \alpha\left\|A x_{2 n+1}-S x_{2 n+1}\right\|\left\|A x_{2 n}-S x_{2 n}\right\| \\
& \quad+\beta\left\|A x_{2 n+1}-T x_{2 n+1}\right\|\left\|A x_{2 n}-T x_{2 n}\right\|+\gamma\left\|x_{2 n+1}-x_{2 n}\right\|^{2} \\
& \leq \alpha\left\|A x_{2 n+1}-A x_{2 n}\right\|\left\|A x_{2 n}-A x_{2 n+1}\right\| \\
& \quad+\beta\left\|A x_{2 n+1}-A x_{2 n}\right\|\left\|A x_{2 n}-A x_{2 n+1}\right\|+\gamma\left\|x_{2 n+1}-x_{2 n}\right\|^{2} \\
& \leq(\alpha+\beta)\left[\frac{\left\|A x_{2 n+1}-A x_{2 n}\right\|^{2}}{2}+\frac{\left\|A x_{2 n}-A x_{2 n-1}\right\|^{2}}{2}\right] \\
& \quad+\gamma\left\|x_{2 n+1}-x_{2 n}\right\|^{2} .
\end{aligned}
$$

This gives

$$
\begin{gathered}
2\left\|A x_{2 n+1}-A x_{2 n}\right\|^{2} \leq(\alpha+\beta)\left[\left\|A x_{2 n+1}-A x_{2 n}\right\|^{2}+\left\|A x_{2 n}-A x_{2 n-1}\right\|^{2}\right] \\
+2 \gamma\left\|x_{2 n+1}-x_{2 n}\right\|^{2}
\end{gathered}
$$

Resulting in

$$
\begin{aligned}
\left\|A x_{2 n+1}-A x_{2 n}\right\|^{2} \leq & \left(\frac{\alpha+\beta}{2-\alpha-\beta}\right)\left\|A x_{2 n}-A x_{2 n-1}\right\|^{2} \\
& +\left(\frac{2 \gamma}{2-\alpha-\beta}\right)\left\|x_{2 n+1}-x_{2 n}\right\|^{2} .
\end{aligned}
$$

Further, observe that

$$
\begin{aligned}
\left\|A x_{2 n}-A x_{2 n-1}\right\|^{2} & \leq \alpha\left\|A x_{2 n}-S x_{2 n}\right\|\left\|A x_{2 n-1}-S x_{2 n-1}\right\| \\
& +\beta\left\|A x_{2 n}-T x_{2 n}\right\| A x_{2 n-1}-T x_{2 n-1}\|+\gamma\| x_{2 n}-x_{2 n-1} \|^{2}
\end{aligned}
$$


Simplifying this using Young's inequality, we get

$$
\begin{aligned}
\left\|A x_{2 n}-A x_{2 n-1}\right\|^{2} & \leq\left(\frac{\alpha+\beta}{2-\alpha-\beta}\right)\left\|A x_{2 n-1}-A x_{2 n-2}\right\|^{2} \\
& +\left(\frac{2 \gamma}{2-\alpha-\beta}\right)\left\|x_{2 n}-x_{2 n-1}\right\|^{2}
\end{aligned}
$$

$$
\begin{aligned}
& \text { Since } \begin{array}{l}
\alpha+\beta+\gamma<1 \\
\frac{\alpha+\beta}{2-\alpha-\beta},
\end{array}, \frac{2 \gamma}{2-\alpha-\beta} \in(0,1)
\end{aligned}
$$

$$
\lambda=\max \left\{\frac{\alpha+\beta}{2-\alpha-\beta}, \frac{2 \gamma}{2-\alpha-\beta}\right\}
$$

Suppose

then $0<\lambda<1$.

From (4) and (5), we conclude that

$$
\begin{aligned}
\left\|A x_{n+1}-A x_{n}\right\|^{2} & \leq \lambda\left[\left\|A x_{n}-A x_{n-1}\right\|^{2}+\left\|A x_{n+1}-A x_{n}\right\|^{2}\right] \\
& \leq \lambda^{2}\left[\left\|A x_{n-1}-A x_{n-2}\right\|^{2}+\left\|A x_{n}-A x_{n-1}\right\|^{2}\right] \\
& +\lambda\left\|A x_{n+1}-A x_{n}\right\|^{2} \\
& \cdots \cdots \cdots \cdots \\
& \leq \lambda^{n}\left[\left\|A x_{1}-A x_{0}\right\|^{2}+\left\|A x_{2}-A x_{1}\right\|^{2}\right]+\varepsilon,
\end{aligned}
$$

for large $n$.

Now, it can be seen that $\left\{A x_{n}\right\}$ is a Cauchy sequence and so it has a limit $x$ in $X$. Since sequences of $\left\{S x_{2 n+1}\right\}$ and $\left\{T x_{2 n}\right\}$ are subsequences of $\left\{A x_{n}\right\}$, they have the same limit $z$. As, $S$ and $A$ are commuting mapping, we can have

$$
\begin{aligned}
S z & =\lim _{n \rightarrow \infty} S A x_{2 n+1} \\
& =\lim _{n \rightarrow \infty} A S x_{2 n+1}=A z
\end{aligned}
$$

Similarly, we get $T z=A z$

This gives us,

$$
T z=A z=S z
$$

It now follows that

$$
\begin{aligned}
\|A z-A A z\|^{2} \leq & \alpha\|A z-S z\|\|A A z-S A z\|+\beta\|A z-T z\|\|A A z-T A z\| \\
& +\gamma\|z-A z\|^{2}
\end{aligned}
$$

In view of (6) and commutativity of $\mathrm{A}$ with $\mathrm{S}$ and $\mathrm{T}$, we find that

$$
\|A A z-T A z\|=\|A A z-A T z\|=\|A A z-A A z\|=0
$$

and

$$
\|A z-S A z\|=\|A z-A A z\| .
$$

Also,

$$
\|A z-T A z\|=\|A z-A A z\| .
$$

Making use of these, we reach at

$$
\|A z-A A z\|^{2} \leq \gamma\|z-A z\|^{2}
$$

Since, ${ }^{\gamma<1}$, we must have $A z=A A z$

Finally, putting $A z=z_{1}$, we have

$$
A z_{1}=A A z=A z=z_{1}
$$

Similarly,

$$
T z_{1}=T A z=A T z=A A z=A z=z_{1}
$$

and

$$
S z_{1}=z_{1}
$$

So, $z l$ is a fixed point of $S, T$, and $A$.

Next, to show uniqueness of this common fixed point, let us suppose that $z 2$ is also a common fixed point of $S, T$, and A other than $z 1$.Then

$$
S z_{2}=z_{2}, T z_{2}=z_{2}, A z_{2}=z_{2}
$$

and also $\left\|z_{1}-z_{2}\right\| \neq 0$.

Hence, it follows that

Thus,

$$
\begin{aligned}
\left\|z_{1}-z_{2}\right\|^{2} & =\left\|A z_{1}-A z_{2}\right\|^{2} \\
\leq & \alpha\left\|A z_{1}-S z_{1}\right\|\left\|A z_{2}-S z_{2}\right\|+\beta\left\|A z_{1}-T z_{1}\right\|\left\|A z_{2}-T z_{2}\right\| \\
& +\gamma\left\|z_{1}-z_{2}\right\|^{2}
\end{aligned}
$$

$$
\left\|z_{1}-z_{2}\right\|^{2}=\gamma\left\|z_{1}-z_{2}\right\|^{2}
$$

which is a contradiction as $\gamma<1$.

Therefore, uniqueness occurs.

Theorem 11: Let $S$ and $T$ be continuous mappings of a Hilbert space $X$ into itself. Then $S$ and $T$ have a common fixed point in $X$ iff there exists a continuous mapping $A$ of $X$ into $S X \cap T X$, which commutes with $S$ and $T$ and satisfies the inequality

$$
\begin{aligned}
\|A x-A y\|^{p / q} \leq & \alpha\|A x-S x\|^{p / q}+\beta\|A y-T y\|^{p / q}+\gamma\|A x-S y\|^{p / q} \\
& +\delta\|A x-T y\|^{p / q}
\end{aligned}
$$

for all $x, y$ in $X$; where $\alpha, \beta, \gamma, \delta$ are non-negative reals with $0<\alpha+\beta+(2 \gamma+2 \delta) 2^{p / q}<1$, where $p$, $q$ are positive real numbers with $p / q<1$

Proof: First of all we prove that the existence of such a mapping $A$ is necessary. For this suppose, $S z=z=T z$ for some $\mathrm{z}$ in $X$.

Let us define a mapping $A$ of $X$ into $X$ by $A x=z$ for all $x$ in $X$. Then clearly, $A$ is continuous mapping of $X$ into $S X \cap T X$. Since, $S x, T x \in X$, for all $x \in X$ and $A x=z$, for all $x \in X$, we get 


$$
A S x=z, S A x=S z=z, A T x=z, T A x=T z=z
$$

Hence, $A$ commutes with $S$ and $T$. Now, for any $\alpha, \beta, \gamma, \delta$ with $0<\alpha+\beta+\gamma+\delta<1$, it is observed that

$$
\begin{aligned}
\|A x-A y\|^{p / q} \leq & \alpha\|A x-S x\|^{p / q}+\beta\|A y-T y\|^{p / q}+\gamma\|A x-S y\|^{p / q} \\
& +\delta\|A x-T y\|^{p / q}
\end{aligned}
$$

which gives

$$
0 \leq \alpha\|A x-S x\|^{p / q}+\beta\|A y-T y\|^{p / q}+\gamma\|A x-S y\|^{p / q}+\delta\|A x-T y\|^{p / q}
$$

for all $x, y$ in $X$. This proves the existence of such a mapping $\mathrm{A}$ is necessary.

To prove sufficient part, sequence $\left\{x_{n}\right\}$ is constructed as follows. Let $x_{0} \in X$ be an arbitrary point.

Since $A X \subset S X$, we choose a point ${ }^{x_{1}}$ in $\mathrm{X}$ such that $S x_{1}=A x_{0}$.

Also, $A X \subset T X$ and hence we can choose $x_{2} \in X$ such that $T x_{2}=A x_{1}$.

Continuing in this way, we get a sequence $\left\{x_{n}\right\}$ as follows:

$$
S x_{2 n-1}=A x_{2 n-2}, T x_{2 n}=A x_{2 n-1} \quad n=1,2,3, \ldots
$$

We proceed to show that $\left\{A x_{n}\right\}$ is a Cauchy sequence. For this we have the inequality,

$$
\begin{aligned}
\left\|A x_{2 n+1}-A x_{2 n}\right\|^{p / q} \leq & \alpha\left\|A x_{2 n+1}-S x_{2 n+1}\right\|^{p / q}+\beta\left\|A x_{2 n}-T x_{2 n}\right\|^{p / q} \\
& +\gamma\left\|A x_{2 n+1}-S x_{2 n}\right\|^{p / q}+\delta\left\|A x_{2 n+1}-T x_{2 n}\right\|^{p / q} \\
= & \alpha\left\|A x_{2 n+1}-A x_{2 n}\right\|^{p / q}+\beta\left\|A x_{2 n}-A x_{2 n-1}\right\|^{p / q} \\
& +\gamma\left\|A x_{2 n+1}-A x_{2 n-1}\right\|^{p / q}+\delta\left\|A x_{2 n+1}-A x_{2 n-1}\right\|^{p / q} \\
& =\alpha\left\|A x_{2 n+1}-A x_{2 n}\right\|^{p / q}+\beta\left\|A x_{2 n}-A x_{2 n-1}\right\|^{p / q} \\
& +(\gamma+\delta)\left\|A x_{2 n+1}-A x_{2 n}+A x_{2 n}-A x_{2 n-1}\right\|^{p / q} \\
\leq & \alpha\left\|A x_{2 n+1}-A x_{2 n}\right\|^{p / q}+\beta\left\|A x_{2 n}-A x_{2 n-1}\right\|^{p / q} \\
& +(\gamma+\delta) 2^{p / q}\left[\left\|A x_{2 n+1}-A x_{2 n}\right\|^{p / q}+\left\|A x_{2 n}-A x_{2 n-1}\right\|^{p / q}\right]
\end{aligned}
$$

because $\|a+b\|^{p} \leq 2^{p}\left(\|a\|^{p}+\|b\|^{p}\right)$ for $0<p<1$. (cf [15]).

which

leads

$\left(1-\alpha-(\gamma+\delta) 2^{p / q}\right)\left\|A x_{2 n+1}-A x_{2 n}\right\|^{p / q} \leq\left(\beta+(\gamma+\delta) 2^{p / q}\right)\left\|A x_{2 n}-A x_{2 n-1}\right\|^{p / q}$

which yields

$\left\|A x_{2 n+1}-A x_{2 n}\right\|^{p / q} \leq\left(\frac{\beta+(\gamma+\delta) 2^{p / q}}{1-\alpha-(\gamma+\delta) 2^{p / q}}\right)\left\|A x_{2 n}-A x_{2 n-1}\right\|^{p / q}$

Similarly we have

$\left\|A x_{2 n}-A x_{2 n-1}\right\|^{p / q} \leq\left(\frac{\beta+(\gamma+\delta) 2^{p / q}}{1-\alpha-(\gamma+\delta) 2^{p / q}}\right)\left\|A x_{2 n-1}-A x_{2 n-2}\right\|^{p / q}$
Hence, one concludes that

$\left\|A x_{n+1}-A x_{n}\right\|^{p / q} \leq\left(\frac{\beta+(\gamma+\delta) 2^{p / q}}{1-\alpha-(\gamma+\delta) 2^{p / q}}\right)\left\|A x_{n}-A x_{n-1}\right\|^{p / q}$

which implies that

$\left\|A x_{n+1}-A x_{n}\right\| \leq\left(\frac{\beta+(\gamma+\delta) 2^{p / q}}{1-\alpha-(\gamma+\delta) 2^{p / q}}\right)^{q / p}\left\|A x_{n}-A x_{n-1}\right\|$

Suppose, now

$$
\lambda=\left(\frac{\beta+(\gamma+\delta) 2^{p / q}}{1-\alpha-(\gamma+\delta) 2^{p / q}}\right)^{q / p}
$$

Then $\mathrm{O}<\lambda<1$ as we have

$$
0<\alpha+\beta+(2 \gamma+2 \delta) 2^{p / q}<1 .
$$

From (7) and (8), we infer that

$$
\begin{aligned}
\left\|A x_{n+1}-A x_{n}\right\| \leq & \lambda\left\|A x_{n}-A x_{n-1}\right\| \\
\leq & \lambda^{2}\left\|A x_{n-1}-A x_{n-2}\right\| \\
& \cdots \cdots \cdots \cdots \\
\leq & \lambda^{n}\left\|A x_{1}-A x_{0}\right\|
\end{aligned}
$$

for large $n$. Now, it can be seen that $\left\{A x_{n}\right\}$ is a Cauchy sequence and so it has a limit $x$ in $X$. Since sequences $\left\{S x_{2 n+1}\right\}$ and $\left\{T x_{2 n}\right\}$ are subsequences of $\left\{A x_{n}\right\}$, they have the same limit z. As, $S$ and $A$ are commuting mapping, we can have

$$
\begin{aligned}
S z & =\lim _{n \rightarrow \infty} S A x_{2 n+1} \\
& =\lim _{n \rightarrow \infty} A S x_{2 n+1}=A z
\end{aligned}
$$

Similarly, we get $T z=A z$

This gives us,

$$
T z=A z=S z
$$

It comes out that

$$
\begin{aligned}
\|A z-A A z\|^{p / q} \leq & \alpha\|A z-S z\|^{p / q}+\beta\|A A z-T A z\|^{p / q} \\
& +\gamma\|A z-S A z\|^{p / q}+\delta\|A z-T A z\|^{p / q}
\end{aligned}
$$

Commutativity of $A$ with $S$ and $T$ gives the following by (9).

$$
\|A A z-T A z\|=\|A A z-A T z\|=\|A A z-A A z\|=0
$$

and

$$
\|A z-S A z\|=\|A z-A A z\|
$$

Also,

$$
\|A z-T A z\|=\|A z-A A z\|
$$

Resorting to these, we arrive at 


$$
\|A z-A A z\|^{p / q} \leq(\gamma+\delta)\|A z-A A z\|^{p / q}
$$

Since, $\gamma+\delta<1$, we must have $A z=A A z$

Finally, putting $A z=z_{1} \quad$, we have $A z_{1}=A A z=A z=z_{1}$

Similarly, $\quad T z_{1}=T A z=A T z=A A z=A z=z_{1}, \quad$ and $S z_{1}=z_{1}$.

So, $z 1$ is a fixed point of $S, T$ and $A$.

Next, to show uniqueness of this common fixed point, let us suppose that $z_{2}$ is also a common fixed point of $S, T$ and $A$ other than $\mathrm{z}_{1}$. Then $S z_{2}=z_{2}, T z_{2}=z_{2}, A z_{2}=z_{2}$ and also $\left\|z_{1}-z_{2}\right\| \neq 0$.

Hence, it follows that

$$
\begin{aligned}
\left\|z_{1}-z_{2}\right\|^{p / q} & =\left\|A z_{1}-A z_{2}\right\|^{p / q} \\
\leq & \alpha\left\|A z_{1}-S z_{1}\right\|^{p / q}+\beta\left\|A z_{2}-T z_{2}\right\|^{p / q} \\
& +\gamma\left\|A z_{1}-S z_{2}\right\|^{p / q}+\delta\left\|A z_{1}-T z_{2}\right\|^{p / q}
\end{aligned}
$$

implies

$$
\left\|z_{1}-z_{2}\right\|^{p / q} \leq(\gamma+\delta)\left\|z_{1}-z_{2}\right\|^{p / q}
$$

which is a contradiction as $\gamma+\delta<1$.

Thus occurs the uniqueness.

Analogously we have the following results in addition.

Theorem 12: Let $S$ and $T$ be continuous mappings of a Hilbert space $X$ into itself. Then $S$ and $T$ have a common fixed point in $X$ iff there exists a continuous mapping $A$ of $X$ into $S X \cap T X$, which commutes with $S$ and $T$ and satisfies the inequality,

$$
\begin{gathered}
\|A x-A y\|^{2} \leq \\
+\|A x-S y\|\|S x-T y\|+\beta\|A x-T y\|\|T x-S y\| \\
+\gamma\|x-y\|^{2}
\end{gathered}
$$

for all $x, y$ in $X$; where $\alpha, \beta, \gamma$ are non-negative real with $2 \alpha+2 \beta+\gamma<1$.

Theorem 13: Let $S$ and $T$ be continuous mappings of a Hilbert space $X$ into itself. Then $S$ and $T$ have a common fixed point in $X$ iff there exists a continuous mapping $A$ of $X$ into $S X \cap T X$, which commutes with $S$ and $T$ and satisfies the inequality,

$$
\begin{aligned}
\|A x-A y\|^{2} \leq & \alpha\|A x-S y\|\|S x-T y\|+\beta\|A x-T y\|\|T x-S y\| \\
& +\gamma\|S x-T y\|^{2}
\end{aligned}
$$

for all $x, y$ in $X$; where $\alpha, \beta, \gamma$ are non-negative real with $\alpha+\beta+\gamma<1$.

Lastly, we have

Theorem 14: Let $S$ and $T$ be continuous mappings of a Hilbert space $X$ into itself. Then $S$ and $T$ have a common fixed point in $X$ iff there exists a continuous mapping $A$ of $X$ into $S X \cap T X$, which commutes with $S$ and $T$ and satisfies the inequality,

$$
\|A x-A y\|^{2} \leq \alpha\|A x-S x\|\|A y-S y\|+\beta\|S x-T x\|^{2}+\gamma\|A x-T x\|\|A y-T y\|
$$

for all $x, y$ in $X$; where $\alpha, \beta, \gamma$ are non-negative reals with $\alpha+\gamma<1$.

\section{CONCLUSION}

The methods adopted in the proofs of fixed point theorems reveal that yet there are various directions in which the Banach's fixed point theorem can be refined and extended retaining the convergence. It is strongly felt that some of the results presented here can be generalized and modified from rate of convergence point of view.

\section{ACKNOWLEDGMENT}

The authors acknowledge with thanks the comments and suggestions of the Reviewers and Editors for improving the presentation of our paper in a more fruitful way.

\section{REFERENCES}

[1] F. E. Browder and W. V. Petryshyn, "Construction of fixed point of non linear mappings in Hilbert space," J. Math. Anal. Appl., vol. 20, pp. 197-228, 1987.

[2] P. V. Koparde and B. B. Waghmode, "On common fixed point theorem for three mappings in Hilbert space," The Math. Ed., vol. 28, no. 1 , pp. 6-9, 1994.

[3] J. Gerald, "Commuting mappings and fixed points," Amer. Math., vol. 83, pp. 261-263, 1976.

[4] F. Brain. "Mapping with a common," Math. Sem. Notes, vol. 7, pp. 81-83, 1979.

[5] R. K. Bisht and R. U. Joshi, "Common fixed point theorems of weakly reciprocal continuous maps," Journal of the Indian Math. Soc., vol. 79, no. 1-4, 2012, 1968

[6] R. Kannan, "Some results on fixed points," Bull. Cal. Math. Soc., vol. 60, pp. 71-76, 1986.

[7] R. Kannan, "Some results on fixed points-II," Amer. Math, vol. 76, pp. 405-408, 1969.

[8] R. P. Pant, "Common fixed points of four mappings," Bull. Cal. Math. Soc., vol. 90, pp. 281-286, 1998.

[9] R. P. Pant, R. K. Bisht, and D. Arora, "Weak reciprocal continuity and fixed point theorems," Ann Univ. Ferrara, vol. 57, pp. 181-190, 2011.

[10] R. P. Pant and R. K. Bisht, "Common fixed point theorems under a new continuity condition," Ann Univ. Ferrara, vol. 58, pp. 127-141, 2012.

[11] W. Takahashi, "A convexity in metric spaces and nonexpansive mappings-I," Kodai Math. Sem. Rep., vol. 22, pp. 142-149, 1970.

[12] M. Moosaei, "Fixed point theorems in convex metric spaces," Fixed Point Theory Appl., pp. 164, 2012.

[13] S. S. Yao and L. H. Qiu, "Hybrid iteration method for fixed points of nonself nonexpansive mapping in real Banach space and its applications," Int. Math., forum 7, no. 6, pp. 251-258, 2012.

[14] G. Abduvalieva and D. S. K.Verbovetskyi, "Fixed point theorems for noncommutative functions," J. Math. Anal. Appl. 401, pp. 436-446, 2013.

[15] H. L. Royden, Real Analysis, PHI, NY, ch. 6, pp.122-123, 1988.

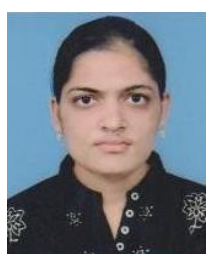

Krishna P. Patel was born on February 10, 1987. She is M.Phil. student in Department of Mathematics, Sardar Patel University, Vallabh Vidhya Nagar, Gujarat, India. She is near to submit her dissertation. She has completed her B.Sc. (2009) from North Gujarat University, Patan, Gujarat, India. M.Sc. (2011) with distinction from Department of Mathematics, Maharaja Sayajirao University, Vadodara, Gujarat, India. She has 1-year teaching experience in Hasmukh Goswami College of Engineering, Vahelal, Ahmedabad, Gujarat, India.

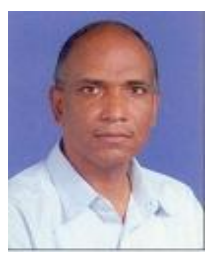

G. M. Deheri is at present attached with Department of Mathematics, Sardar Patel University, Vallabh Vidhya Nagar, Gujarat, India. To his credit he has a large experience of around 27-years in teaching at the post graduate level. He has published more than 150 research papers in various International and National Journals. His research interests are in Functional Analysis and Tribology research. He has also won a few awards for the excellence in research. 